\title{
Human embryo research deserves public support
}

To the editor - Human embryo research which, in the context of this discussion, might more properly be called research on in vitro fertilization (IVF) and on the preimplantated embryo, is essential to the diagnosis, treatment and prevention of human disease and, when pursued according to ethical guidelines, deserves public support.

Early in 1994, the National Institutes of Health (NIH) established a Human Embryo Research Panel, comprised of scientists, ethicists, and lawyers, to review the key issues related to this research and to propose appropriate research guidelines. After weighing the ethical issues that pertain to embryo research, and after considering the opinions of the public at large, the embryo panel made recommendations to the director of the NIH on research as it relates to IVF and the preimplantated embryo that have significant implications for the future understanding and treatment of infertility, the diagnosis and prevention of a range of genetic and developmental dis- orders, and prevention of environmental toxic effects in newborns, among others.

The entire topic is subject to inflammatory rhetoric and scary fantasies. People imagine embryos being created in petri dishes only to be tortured and destroyed. Nothing is further from the truth. The present situation reminds me of the fear that accompanied the early research with recombinant DNA technology and socalled genetic engineering. Images of monsters coming from the sewers never materialized, because the scary image put forward by opponents of scientific progress was never real in the first place. We in the United States (and elsewhere) are still confronted by concerns about somatic cell gene therapy, although open debate and early successes in several diseases have made the public more informed and, therefore, more supportive of gene therapy in human subjects.

I hope the same can soon be said of IVF and preimplantation embryo research. It is incumbent on us in science to do everything we can to discuss with the public in clear, honest and open language just what this research is all about. We need to insure that the proposed research in this field is subject to full and open debate as suggested by the NIH Director's Advisory Council on 2 December 1994.

We need this research to prevent terrible human suffering. I hope that with the cautious but forward-thinking guidelines proposed by the embryo research panel, it will be possible for the NIH to proceed with support of research in this important area.

\section{William N. Kelley}

\author{
Chief Executive Officer \\ University of Pennsylvania, \\ School of Medicine, \\ Philadelphia, Pennsylvania \\ Dean, School of Medicine. \\ Philadelphia, Pennsylvania
}

\section{Hypersensitivity to nonsteroidal anti-inflammatory drugs}

To the editor - Nonsteroidal anti-inflammatory drugs (NSAIDs) are a widely used class of compounds that are prescribed as analgesic, antipyretic and antiinflammatory agents ${ }^{1}$. NSAIDs can elicit potentially fatal hypersensitivity reactions (including anaphylaxis, bronchospasm and hepatotoxicity ${ }^{2-7}$ suggestive of an IgE or IgG mediated immune response. Patients allergic to one NSAID are generally advised against taking another of these agents because there is a degree of cross-reactivity with respect to the allergic-like reactions.Many of these drugs possess a carboxylic acid moiety that can be conjugated with glucuronic acid to form an acyl glucuronide.These glucuronide conjugates have been shown to bind covalently to proteins both in vitro and in vivo ${ }^{8-10}$.Tandem mass spectrometry studies have illustrated that protein adducts formed between tolmetin glucuronide and albumin contain tolmetin bound to some amino acid residues via a glucuronic acid bridge and tolmetin bound directly to other residues $^{8,11}$. We have hypothesized that the allergic-like reactions and the organ toxicities may occur via an immune based mechanism ${ }^{12}$ whereby an acyl glucuronide metabolite of acidic NSAIDs acts as a hapten upon covalent binding to a protein ${ }^{13}$. We can now demonstrate that immunization with a mouse serum albumin conjugate of tolmetin glucuronide stimulated an antibody response in mice. Antibodies specific for tolmetin and tolmetin glucuronide were generated and these antibodies cross-reacted with other NSAIDs and their glucuronides. This suggests that hypersensitivity to NSAIDs is initiated by an immune response to drug- or drug-glucuronide-protein conjugates and that cross-reactive hypersensitivity is due to cross-reacting antibodies.

Mouse serum albumin (MSA), chosen as a model self-protein, was reacted with tolmetin glucuronide (TG) to produce a TG-MSA conjugate. For control experiments, MSA was incubated with unmetabolized tolmetin ( $T$ ) under the same conditions used to produce the TG-MSA conjugate. Twelve mice received the TGMSA conjugate and a further $\mathbf{1 2}$ mice received unmodified MSA.

Antibodies binding to tolmetin glucuronide-human serum albumin conjugate (TG-HSA) were detected in $25 \%$ of the mice immunized with the TG-MSA conjugate.TG-specific titres were 1:8000 after four immunizations. No conjugate specific antibodies were observed in any of the mice immunized with unconjugated MSA. To confirm that the antibodies reacting with TG-HSA were reactive with the TG-hapten, the active antisera were incubated with soluble TG-MSA and then retested on TG-HSA. This resulted in complete blocking of the antibody binding (data not shown) whereas preincubation with MSA alone had no effect. These results indicate that TG-MSA conjugate is immunogenic in mice. Both the selectivity and specificity of the immune response in mice mirrors the human hypersensitivity reaction to NSAIDs. Only a small fraction of the treated human pop- 